\title{
Combined free and forced convection flow in a rotating channel with arbitrary conducting walls
}

\author{
G.S. Seth $^{1 *}$, N. Mahto ${ }^{2}$, Md. S. Ansari ${ }^{1}$ and R. Nandkeolyar ${ }^{1}$ \\ ${ }^{1}$ Department of Applied Mathematics, Indian School of Mines, Dhanbad-826004, INDIA \\ ${ }^{2}$ Department of Mathematics, R.S.P. College, Jharia, Dhanbad, INDIA \\ *Corresponding Author: e-mail: gsseth_ism@yahoo.com, Tel +91-326-2235421, Fax.+91-326-2296619
}

\begin{abstract}
Combined free and forced convection flow of a viscous incompressible electrically conducting fluid in a rotating channel is studied. Analytical solution for the velocity and induced magnetic field is obtained in closed form. Asymptotic behavior of the solution for the velocity and induced magnetic field is analyzed for large values of magnetic parameter $M^{2}$ and rotation parameter $K^{2}$ to gain some physical insight into the flow pattern. The expressions for the shear stress and critical Grashof number at both the plates and mass flow rates are also derived. Numerical values of rate of heat transfer at both the plates are obtained with the help of MATLAB software. The numerical values of velocity and induced magnetic field are displayed graphically whereas that of shear stress at the upper plate, mass flow rate, critical Grashof number and rate of heat transfer at both the plates are presented in tabular form for various values of flow parameters.
\end{abstract}

Keywords: Wall conductance, free and forced convection, magnetic field, rotation, Ekman-Hartmann boundary layer, modified Ekman boundary layer.

\section{Introduction}

The study of hydromagnetic flow of a viscous incompressible electrically conducting fluid in a rotating medium is of considerable importance due to occurrence of various natural phenomena and for its application in various technological situations which are directly governed by the action of Coriolis and magnetic forces. In order to increase basic understanding of such fluid flows, several researchers including Hide and Roberts (1960), Nanda and Mohanty (1971), Soundalgekar and Pop (1973), Soundalgekar and Gupta (1975), Debnath (1974,1975), Acheson (1975), Mazumder (1977), Datta and Jana (1977), Seth et al (1982, 2009, 2010), Raptis and Singh (1986), Singh et al (1994), Ghosh (1993), Nagy and Demendy (1995), Seth and Banerjee (1996), Ghosh and Bhattacharjee (2000), Singh (2000), Ghosh and Pop (2003, 2004), Hayat et al. (2004a, 2004b, 2004c) Seth and Singh (2008), Seth and Ansari (2009), Das et al (2009), Guria et al. (2009) investigated the problems of hydromagnetic flow in rotating medium under different conditions and configurations. Mazumder (1977) studied steady flow of a viscous incompressible electrically conducting fluid in a rotating channel with arbitrary conducting walls. Mazumder (1977) also investigated the heat transfer characteristics of the flow due to forced convection taking viscous and Joule dissipations into account. It is well known that the fluids with low Prandtl number are electrically conducting apart from being more sensitive to the gravitational field than fluids with high Prandtl number. Hence the interplay of buoyancy force with the electromagnetic force determines the ultimate behaviour of an electrically conducting fluid with low Prandtl number under a transverse magnetic field. Keeping in view this fact, Seth and Banerjee (1996), Ghosh and Bhattacharjee (2000), Seth and Singh (2008) and Seth and Ansari (2009) studied combined free and forced convection flow of a viscous incompressible electrically conducting fluid in a rotating channel considering different aspects of the problem. In all these investigations the walls of the channel are considered either non-conducting or perfectly conducting.

The purpose of the present paper is to study combined free and forced convection flow of a viscous incompressible electrically conducting fluid in a rotating channel with arbitrary conducting walls, in the presence of a transverse magnetic field, under the action of a uniform pressure gradient. Analytical solution for the velocity and induced magnetic field is obtained in closed form. 
The solution for the velocity and induced magnetic field, in dimensionless form, contains four pertinent flow parameters, namely, $M^{2}$ (square of Hartmann number), $K^{2}$ (rotation parameter which is the reciprocal of Ekman number), G (Grashof number) and $\phi_{1}$ and $\phi_{2}$ (wall conductance ratios). The asymptotic behavior of the solution is analyzed for large values of $M^{2}$ and $K^{2}$ to gain some physical insight into the flow pattern. The expressions for the shear stress at both the plates due to the primary and secondary flows, mass flow rates and Critical Grashof number at both the plates due to the primary as well as secondary flows are obtained. Heat transfer characteristics of the flow is considered taking viscous and Joule dissipations into account when the plates of the channel are heated or cooled due to uniform temperature gradient. The numerical values of the rate of heat transfer at both the plates are obtained with the help of MATLAB software.

To study the effects of magnetic field, rotation, free convection and wall conductance profiles of velocity are drawn versus channel width variable $\eta$ for various values of $M^{2}, K^{2}, G$ and $\phi=\phi_{1}+\phi_{2}$ while the numerical values of induced magnetic field are depicted graphically versus $\eta$ for different values of $M^{2}, K^{2}, G, \phi_{1}$ and $\phi_{2}$. The numerical values of the primary and secondary shear stress components at the upper plate $\eta=1$ and rate of heat transfer at both the plates are presented in tabular form for various values of $M^{2}, K^{2}, G$ and $\phi$ whereas that of mass flow rates and critical Grashof number are given in tables for different values of $M^{2}, K^{2}$ and $\phi$.

\section{Formulation of the problem and its solution}

Consider combined free and forced convection flow of a viscous incompressible electrically conducting fluid between two horizontal parallel finitely thick arbitrary conducting plates $z= \pm L$ in the presence of a uniform transverse magnetic field $H_{o}$ applied parallel to $z$-axis. The fluid and channel rotate in unison with a uniform angular velocity $\Omega$ about $z$ - axis and fluid flow within the channel is induced due to uniform pressure gradient applied in $x$-direction. The plates of the channel are cooled or heated by uniform temperature gradient acting along $x$-direction so that temperature varies linearly along the plates. Since plates are infinite along $x$ - and $y$-directions and flow is assumed to be steady and fully developed, all physical quantities, except pressure and temperature, depend on $z$ only. Geometry of the problem is presented in Fig. 1.

The fluid velocity $\vec{q}$ and induced magnetic field $\vec{H}$ are assumed as

$$
\vec{q}=\left(u^{\prime}, v^{\prime}, 0\right), \quad \vec{H}=\left(H_{x}{ }^{\prime}, H_{y}{ }^{\prime}, H_{o}\right) .
$$

This assumption is compatible with the fundamental equations of magnetohydrodynamics in a rotating medium.

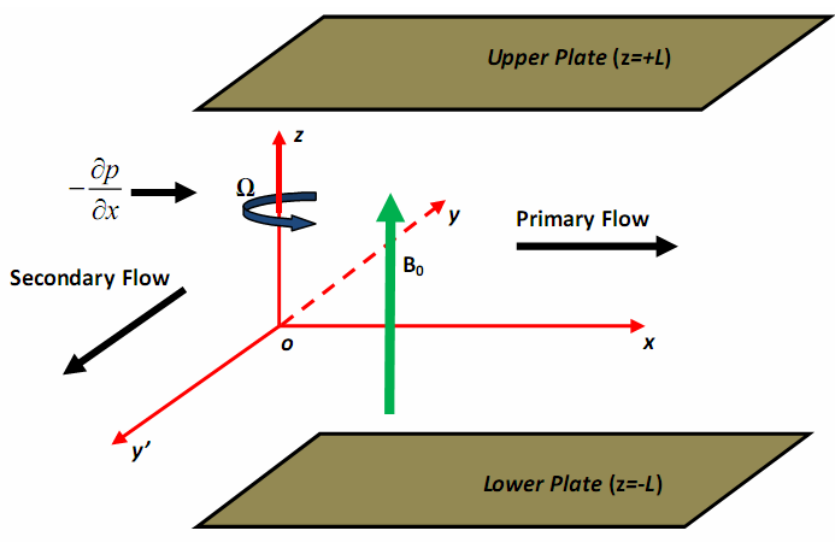

Fig. 1. Geometry of the Problem

Under the above assumptions equation of motion and induction equation for the magnetic field reduce to

$$
\begin{gathered}
-2 \Omega v^{\prime}=-\frac{1}{\rho} \frac{\partial p}{\partial x}+\frac{\mu_{e} H_{O}}{\rho} \frac{d H_{X}^{\prime}}{d z}+v \frac{d^{2} u^{\prime}}{d z^{2}}, \\
2 \Omega u^{\prime}=\frac{\mu_{e} H_{0}}{\rho} \frac{d H_{y}^{\prime}}{d z}+v \frac{d^{2} v^{\prime}}{d z^{2}},
\end{gathered}
$$




$$
\begin{aligned}
& 0=-\frac{1}{\rho} \frac{\partial p}{\partial z}-g\left[1-\beta^{\prime}\left(T^{\prime}-T_{O}\right)\right]-\frac{\mu_{e}}{\rho}\left(H_{X^{\prime}} \frac{d H_{X}{ }^{\prime}}{d z}+H_{y^{\prime}} \frac{d H_{y^{\prime}}}{d z}\right), \\
& -\frac{d^{2} H_{X}{ }^{\prime}}{d z^{2}}=\sigma \mu_{e} H_{O} \frac{d u^{\prime}}{d z}, \\
& -\frac{d^{2} H_{y^{\prime}}}{d z^{2}}=\sigma \mu_{e} H_{O} \frac{d v^{\prime}}{d z},
\end{aligned}
$$

where $p, T_{0}, \rho, v, \mu_{e}, \sigma, \beta^{\prime}$ and $\mathrm{g}$ are, respectively, modified hydromagnetic pressure including centrifugal force, temperature in reference state, density, kinematic coefficient of viscosity, magnetic permeability, electrical conductivity, coefficient of thermal expansion and acceleration due to gravity.

Since plates of the channel are cooled or heated by uniform temperature gradient acting along $x$-direction so the fluid temperature is considered as

$$
T^{\prime}-T_{O}=A_{1} X+\theta_{1}(z),
$$

where $T^{\prime}, \mathrm{A}_{1}$ and $\theta_{1}(\mathrm{z})$ are, respectively, fluid temperature, uniform temperature gradient acting along $\mathrm{x}$ - direction and arbitrary function of $\mathrm{z}$.

Eqn. (4), after integration, is given by

$$
\frac{p}{\rho}=-g z-\frac{\mu_{e}}{2 \rho}\left(H_{X}{ }^{2}+H_{y}{ }^{2}+H_{O}{ }^{2}\right)+g \beta^{\prime} \int\left(T^{\prime}-T_{O}\right) d z+K_{1} x,
$$

where $\mathrm{K}_{1}$ is the uniform pressure gradient acting along $\mathrm{x}$-direction with which fluid flow is generated within the channel.

Eqn. (2), with the help of (7) and (8), reduces to

$$
-2 \Omega v^{\prime}=-g \beta A_{1} z-K_{1}+\frac{\mu_{e} H_{o}}{\rho} \frac{d H_{x}{ }^{\prime}}{d z}+v \frac{d^{2} u}{d z^{2}} \text {. }
$$

The boundary conditions for the velocity field are

$$
u^{\prime}=v^{\prime}=0 \quad \text { at } z= \pm L \text {. }
$$

It is assumed that the plates of the channel are of finite thickness and of arbitrary electrical conductivity, the boundary conditions for the magnetic field (Mazumder (1977)) are

$$
\left.\begin{array}{c}
\frac{d H_{x}{ }^{\prime}}{d z}+\frac{\sigma H_{x}{ }^{\prime}}{\sigma_{1} h_{1}}=0 ; \frac{d H_{y}{ }^{\prime}}{d z}+\frac{\sigma H_{y}{ }^{\prime}}{\sigma_{1} h_{1}}=0 \text { at } z=L, \\
\frac{d H_{x}{ }^{\prime}}{d z}-\frac{\sigma H_{x}{ }^{\prime}}{\sigma_{2} h_{2}}=0 ; \frac{d H_{y}{ }^{\prime}}{d z}-\frac{\sigma H_{y}{ }^{\prime}}{\sigma_{2} h_{2}}=0 \text { at } z=-L,
\end{array}\right\}
$$

where $\sigma_{1}, h_{1}$ and $\sigma_{2}, h_{2}$ are, respectively, electrical conductivity and thickness of the upper and lower plates.

Introducing non-dimensional variables

$$
\eta=z / L, u=u^{\prime} L / v, v=v^{\prime} L / v, H_{x}=H_{x}{ }^{\prime} / \sigma \mu_{e} v H_{o} \text { and } H_{y}=H_{y}{ }^{\prime} / \sigma \mu_{e} v H_{o},
$$

the eqns. (3), (5), (6) and (9), in dimensionless form, are given by

$$
\begin{aligned}
& \frac{d^{2} u}{d \eta^{2}}+M^{2} \frac{d H_{x}}{d \eta}+2 K^{2} v=G \eta+R, \\
& \frac{d^{2} v}{d \eta^{2}}+M^{2} \frac{d H_{y}}{d \eta}-2 K^{2} u=0, \\
& -\frac{d^{2} H_{x}}{d \eta^{2}}=\frac{d u}{d \eta}, \\
& -\frac{d^{2} H_{y}}{d \eta^{2}}=\frac{d v}{d \eta},
\end{aligned}
$$


where $M^{2}=\mu_{e}{ }^{2} H_{O}{ }^{2} L^{2}(\sigma / \rho v)$ is magnetic parameter which is square of Hartmann number, $K^{2}=\Omega L^{2} / v$ is rotation parameter which is the reciprocal of Ekman number, $G=g \beta^{\prime} A_{1} L^{4} / v^{2}$ is Grashof number and $R=L^{3} K_{1} / v^{2}$ is non-dimensional pressure gradient.

It is noticed from equation (7) that positive or negative value of $A_{1}$ corresponds to heating or cooling of channel walls along $x$ direction. Therefore, it follows from the definition of $\mathrm{G}$ that $\mathrm{G}>0$ or $\mathrm{G}<0$ according as the channel walls are heated or cooled in axial direction.

The boundary conditions (10) and (11), in dimensionless form, become

$$
u=v=0 \text { at } \eta= \pm 1 \text {, }
$$

$$
\left.\begin{array}{l}
\frac{d H_{x}}{d \eta}+\frac{H_{x}}{\phi_{1}}=0 ; \frac{d H_{y}}{d \eta}+\frac{H_{y}}{\phi_{1}}=0 \text { at } \eta=1, \\
\frac{d H_{x}}{d \eta}-\frac{H_{x}}{\phi_{2}}=0 ; \frac{d H_{y}}{d \eta}-\frac{H_{y}}{\phi_{2}}=0 \text { at } \eta=-1,
\end{array}\right\}
$$

where $\phi_{1}=\sigma_{1} h_{1} / \sigma L$ and $\phi_{2}=\sigma_{2} h_{2} / \sigma L$ are dimensionless wall conductance ratios for the upper and lower plates respectively.

The eqns. (13) to (16) are presented in compact form as

$$
\begin{aligned}
& \frac{d^{2} F}{d \eta^{2}}+M^{2} \frac{d H}{d \eta}-2 i K^{2} F=G \eta+R, \\
& \frac{d^{2} H}{d \eta^{2}}+\frac{d F}{d \eta}=0,
\end{aligned}
$$

where $F=u+i v$ and $H=H_{x}+i H_{y}$.

The boundary conditions (17) and (18) become

$$
\begin{aligned}
& \mathrm{F}=0 \quad \text { at } \eta= \pm 1 \\
& \frac{d H}{d \eta}+\frac{H}{\phi_{1}}=0 \quad \text { at } \eta=1, \quad \frac{d H}{d \eta}-\frac{H}{\phi_{2}}=0 \quad \text { at } \eta=-1 .
\end{aligned}
$$

The solution of eqns. (19) and (20) subject to the boundary conditions (21) and (22) are given by

$$
\begin{aligned}
& F(\eta)=C\left(1-\frac{\cosh \lambda \eta}{\cosh \lambda}\right)+\frac{G}{\lambda^{2}}\left(\frac{\sinh \lambda \eta}{\sinh \lambda}-\eta\right), \\
& H(\eta)=C\left(\frac{\sinh \lambda \eta}{\sinh \lambda}-\eta\right)+\frac{G}{\lambda^{2}}\left(\frac{1}{2} \eta^{2}-\frac{\cosh \lambda \eta}{\lambda \sinh \lambda}\right)-C_{1} \eta+C_{2},
\end{aligned}
$$

where $\lambda=\alpha+i \beta$,

$$
\begin{aligned}
& C=\frac{R \lambda(\phi+2)}{\left[2 M^{2}(\lambda-\tanh \lambda)-(\phi+2) \lambda^{3}\right]}, \\
& \phi=\phi_{1}+\phi_{2},
\end{aligned}
$$




$$
\begin{aligned}
& C_{1}=\frac{2 R(\lambda-\tanh \lambda)}{2 M^{2}(\tanh \lambda-\lambda)+(\phi+2) \lambda^{3}}, \\
& C_{2}=\frac{1}{2} C_{1}\left(\phi_{1}-\phi_{2}\right)-\frac{G}{2 \lambda^{3}}(\lambda-2 \operatorname{coth} \lambda), \\
& \alpha, \beta=\frac{1}{\sqrt{2}}\left[\left(M^{4}+4 K^{4}\right)^{\frac{1}{2}} \pm M^{2}\right]^{\frac{1}{2}} .
\end{aligned}
$$

\subsection{Shear stress at the upper and lower plates}

The non-dimensional shear stress components $\tau_{x}$ and $\tau_{y}$ at the upper and lower plates, due to the primary and secondary flows respectively, are given by

$$
\left(\tau_{x}+i \tau_{y}\right)_{\eta= \pm 1}=\mp \frac{C \lambda \sinh (\lambda \eta)}{\cosh (\lambda)}+\frac{G}{\lambda^{2}}\left(\frac{\lambda \cosh (\lambda \eta)}{\sinh (\lambda)}-1\right) .
$$

\subsection{Critical Grashof number}

The Critical Grashof numbers $\mathrm{G}_{\mathrm{cx}}$ and $\mathrm{G}_{\mathrm{cy}}$, for which shear stress at the plates due to the primary and secondary flows respectively vanishes, are given by

$\left(G_{c x}+i G_{c y}\right)_{\eta= \pm 1}= \pm \frac{C \lambda^{3} \sinh ^{2}(\lambda)}{\cosh (\lambda)[\lambda \cosh (\lambda)-\sinh (\lambda)]}$.

\subsection{Mass flow rate}

The non-dimensional mass flow rates $\mathrm{Q}_{\mathrm{x}} / \rho v$ and $\mathrm{Q}_{\mathrm{y}} / \rho v$, in the primary and secondary flow directions respectively, are given by

$$
\left(\frac{Q_{x}+i Q_{y}}{\rho v}\right)=\frac{1}{2} \frac{C\left(1+2 \lambda e^{\lambda} \cosh (\lambda)-\left(e^{\lambda}\right)^{2}\right)}{\lambda e^{\lambda} \cos (\lambda)} .
$$

\section{Asymptotic Solutions}

Now asymptotic behavior of the solution given by (23) and (24) is to be discussed to gain some physical insight into the flow pattern.

Case I: $M^{2}>>1$ and $K^{2}>>1$

When both $M^{2}$ and $K^{2}$ are large, boundary layer type flow is expected. For the boundary layer flow adjacent to the upper plate $\eta=1$, introducing boundary layer coordinate $\xi=1-\eta$, the asymptotic solution for the velocity and induced magnetic field are obtained from (23) and (24) which are presented as

$$
\begin{aligned}
& u=\frac{1}{\left(\alpha^{2}+\beta^{2}\right)^{2}}\left[\left(\alpha^{2}-\beta^{2}\right)\{G(\xi-1)-R\}+(G+R) e^{-\alpha \xi}\left\{\left(\alpha^{2}-\beta^{2}\right) \cos \beta \xi-2 \alpha \beta \sin \beta \xi\right\},\right. \\
& v=\frac{1}{\left(\alpha^{2}+\beta^{2}\right)^{2}}\left[2 \alpha \beta\{R-G(\xi-1)\}-(G+R) e^{-\alpha \xi}\left\{\left(\alpha^{2}-\beta^{2}\right) \sin \beta \xi+2 \alpha \beta \cos \beta \xi\right\}\right], \\
& H_{x}=\left\{-\frac{G}{2} \xi(2-\xi)+R\left(1-\xi-\phi_{3}\right)\right\} \delta-\frac{e^{-\alpha \xi}}{\left(\alpha^{2}+\beta^{2}\right)}(G+R)[(\alpha \delta+\beta \gamma) \cos \beta \xi+
\end{aligned}
$$




$$
\begin{aligned}
& +(\beta \delta-\alpha \gamma) \sin \beta \xi]+\frac{1}{\left(\alpha^{2}+\beta^{2}\right)}(\alpha \delta+\beta \gamma)\left(G+R \phi_{3}\right), \\
H_{y}= & \left\{-\frac{G}{2} \xi(2-\xi)+R\left(1-\xi-\phi_{3}\right)\right\} \gamma-\frac{(G+R)}{\left(\alpha^{2}+\beta^{2}\right)} e^{-\alpha \xi}[(\alpha \delta+\beta \gamma) \sin \beta \xi+ \\
& +(\beta \delta-\alpha \gamma) \cos \beta \xi]+\frac{1}{\left(\alpha^{2}+\beta^{2}\right)}(\alpha \gamma-\beta \delta)\left(G+R \phi_{3}\right),
\end{aligned}
$$

where

$$
\delta=\frac{\left(\alpha^{2}-\beta^{2}\right)}{\left(\alpha^{2}+\beta^{2}\right)^{2}}, \gamma=-\frac{2 \alpha \beta}{\left(\alpha^{2}+\beta^{2}\right)^{2}}, \phi_{3}=\frac{2(1-\xi)+\left(\phi_{2}-\phi_{1}\right)}{(\phi+2)} .
$$

It is evident from the expressions (29) to (32) that flow is divided into two regions, namely, boundary layer region and central core region. The boundary layer region is confined to the boundary layer of thickness $O(1 / \alpha)$ which arises adjacent to the upper plate of the channel. This layer may be recognized as modified Ekman-Hartmann boundary layer and can be viewed as either a classical Ekman boundary layer modified by magnetic field or a classical Hartmann boundary layer modified by rotation.

For $M^{2}>>K^{2}$ this boundary layer reduces to the Hartmann boundary layer and for $M^{2}<<K^{2}$ it becomes Ekman boundary layer. A similar type of boundary layer appears near the lower plate. The exponential terms in (29) to (32) damp out quickly as $\xi$ increases. When $\xi \geq 1 / \alpha$, i.e. in the central core region, (29) to (32) reduce to

$$
\begin{aligned}
& u \approx \frac{1}{\left(\alpha^{2}+\beta^{2}\right)^{2}}\left[\left(\alpha^{2}-\beta^{2}\right)\{G(\xi-1)-R\}\right], \quad v \approx \frac{2 \alpha \beta}{\left(\alpha^{2}+\beta^{2}\right)^{2}}[R-G(\xi-1)], \\
& H_{x} \approx\left\{-\frac{G}{2} \xi(2-\xi)+R\left(1-\xi-\phi_{3}\right)\right\} \delta+\frac{(\alpha \delta+\beta \gamma)\left(G+R \phi_{3}\right)}{\left(\alpha^{2}+\beta^{2}\right)}, \\
& H_{y} \approx\left\{-\frac{G}{2} \xi(2-\xi)+R\left(1-\xi-\phi_{3}\right)\right\} \gamma+\frac{(\alpha \gamma-\beta \delta)\left(G+R \phi_{3}\right)}{\left(\alpha^{2}+\beta^{2}\right)} .
\end{aligned}
$$

Expressions (34) to (36) reveal that, in central core region, fluid flows in both the primary as well as secondary flow directions and has considerable effects of rotation and magnetic field. The effect of free convection on the velocity become insignificant near the central line of the channel (i.e. $\eta=0$ ). The fluid velocity varies linearly with $\eta$ in both the directions. The induced magnetic field components $H_{x}$ and $H_{y}$ have considerable effects of rotation, magnetic field, wall conductance and free convection. Also $H_{x}$ and $H_{y}$ vary with $\eta$ but the variation is non- linear due to the presence of free convection. In the absence of free convection fluid flow is uniform in the central core region while induced magnetic field varies linearly with $\eta$.

Case II: $K^{2}>>1$ and $M^{2} \sim O(1)$

In this case also fluid flow becomes boundary layer type. For the boundary layer flow near the upper plate, the expressions for the velocity and induced magnetic field, derived from (23) and (24), are given by

$$
\begin{aligned}
& u=-\frac{(R+G)}{2 K^{2}} e^{-\alpha_{1} \xi} \sin \beta_{1} \xi, \\
& v=\frac{1}{2 K^{2}}\left[(R+G)\left(1-e^{-\alpha_{1} \xi} \cos \beta_{1} \xi\right)-G \xi\right] \text {, } \\
& H_{x}=-\frac{1}{4 K^{3}}\left[\left(R \phi_{3}+G\right)-\sqrt{2}(R+G) e^{-\alpha_{1} \xi} \cos \left(\beta_{1} \xi-\frac{\pi}{4}\right)\right] \text {, } \\
& H_{y}=-\frac{1}{4 K^{2}}\left[2 R\left\{\phi_{3}+(1-\xi)\right\}-G \xi(2-\xi)+\frac{1}{k}\left\{\left(R \phi_{3}+G\right)+\sqrt{2}(R+G) \times e^{-\alpha_{1} \xi} \sin \left(\beta_{1} \xi-\frac{\pi}{4}\right)\right\}\right] \text {, }
\end{aligned}
$$

where

$$
\alpha_{1}=K\left[1+\frac{M^{2}}{4 K^{2}}\right], \beta_{1}=K\left[1-\frac{M^{2}}{4 K^{2}}\right]
$$


The expressions (37) to (40) show that the fluid flow is divided into two regions viz. boundary layer region and central core region. The boundary layer region is confined to the boundary layer of thickness $\mathrm{O}\left(1 / \alpha_{1}\right)$ which appears near the upper plate. This boundary layer may be identified as modified hydromagnetic Ekman boundary layer and can be viewed as a classical Ekman boundary layer modified by magnetic field. A similar type of boundary layer appears near the lower plate. The thickness of the boundary layer decreases with the increase in either $K^{2}$ or $M^{2}$. In the central core region, the expressions (37) to (40) reduce to

$$
\begin{aligned}
& u \approx 0, v \approx \frac{1}{2 K^{2}}(R+G \eta), \\
& H_{x} \approx-\frac{1}{4 K^{3}}\left(R \phi_{3}+G\right), \\
& H_{y} \approx-\frac{1}{4 K^{2}}\left[\left\{2 R\left(\phi_{3}+\eta\right)-G\left(1-\eta^{2}\right)\right\}+\frac{1}{K}\left(R \phi_{3}+G\right)\right]
\end{aligned}
$$

It is evident from the expressions (42) to (44) that, in the central core region, fluid flows in a direction normal to the directions of applied pressure gradient and axis of rotation. Fluid velocity is unaffected by the magnetic field and varies linearly with $\eta$. The effect of free convection on fluid flow is insignificant near central line of the channel. The induced magnetic field persists in both the directions due to free convection and wall conductance in this region and has considerable effects of rotation, wall conductance and free convection. Induced magnetic field $H_{x}$ varies linearly with $\eta$ whereas variation in induced magnetic field $H_{y}$ is non-linear with $\eta$ due to the presence of free convection.

\section{Case III: $M^{2}>>1$ and $K^{2} \sim O(1)$}

In this case also boundary layer type flow is expected. For the boundary layer flow near the upper plate, the expressions for the velocity and induced magnetic field, obtained from (23) and (24), are given by

$$
\begin{aligned}
& u=\frac{(R+G)}{M^{2}}\left\{e^{-M \xi} \cos \beta_{2} \xi-1\right\}+\frac{G \xi}{M^{2}}, \\
& v=\frac{(R+G)}{M^{2}} e^{-M \xi} \sin \beta_{2} \xi, \\
& H_{x}=-\frac{1}{2 M^{2}}\left[2 R\left(1-\frac{1}{M}\right) \phi_{3}+G\left(1-\frac{2}{M}\right)-\{2 R+G(1-\xi)\}(1-\xi)+\frac{2}{M}(R+G) e^{-M \xi} \cos \beta_{2} \xi\right] \text {, } \\
& H_{y}=\frac{(R+G)}{M^{3}} e^{-M \xi} \sin \beta_{2} \xi,
\end{aligned}
$$

where

$$
\beta_{2}=K^{2} / M .
$$

It is evident from the expressions (45) to (48) that fluid flow is divided into two regions, namely, boundary layer region and central core region. The boundary layer region is confined to Hartmann boundary layer of thickness $\mathrm{O}(1 / \mathrm{M})$ which arises near the upper plate of the channel. A similar type of boundary layer appears adjacent to the lower plate. In the central core region, the expressions (45) to (48) reduce to

$$
\begin{aligned}
& u \approx-\frac{1}{M^{2}}(G \eta+R), v \approx 0, \\
& H_{x} \approx-\frac{1}{2 M^{2}}\left[2 R\left(1-\frac{1}{M}\right) \phi_{3}+G\left(1-\frac{2}{M}\right)-(2 R+G \eta) \eta\right], .
\end{aligned}
$$

$H_{y} \approx 0$.

The expressions (50) to (52) reveal that, in the central core region, the velocity and induced magnetic field vanish away in the secondary flow direction while it persist in the primary flow direction and are unaffected by rotation. The primary velocity $u$ varies linearly with $\eta$ and the effect of free convection on it is insignificant near the central line of the channel. The variation in induced magnetic field $H_{x}$ is non-linear with $\eta$ due to the presence of free convection and it has considerable effects of magnetic field, wall conductance and free convection. 


\section{Heat Transfer Characteristics}

Energy equation for steady fully developed flow of a viscous incompressible electrically conducting fluid taking viscous and Joule dissipations into account is

$$
u^{\prime} \frac{\partial\left(T^{\prime}-T_{o}\right)}{\partial x}=\frac{k}{\rho c_{p}} \frac{\partial^{2}\left(T^{\prime}-T_{0}\right)}{\partial z^{2}}+\frac{v}{c_{p}}\left[\left(\frac{d u^{\prime}}{d z}\right)^{2}+\left(\frac{d v^{\prime}}{d z}\right)^{2}\right]+\frac{1}{\sigma \rho c_{p}}\left[\left(\frac{d H_{x}{ }^{\prime}}{d z}\right)^{2}+\left(\frac{d H_{y}{ }^{\prime}}{d z}\right)^{2}\right],
$$

where $k$ and $C_{p}$ are, respectively, thermal conductivity and specific heat at constant pressure.

Since plates $Z= \pm L$ are cooled or heated by a uniform temperature gradient $\mathrm{A}_{1}$ along $\mathrm{x}$ - direction, therefore, boundary conditions for fluid temperature are assumed as

$\left.\begin{array}{l}T^{\prime}=T_{o}+A_{1} X+\theta_{1 w_{1}} \text { at } z=L \\ T^{\prime}=T_{o}+A_{1} X+\theta_{1 w_{2}} \text { at } z=-L,\end{array}\right\}$

where $\theta_{1 w_{1}}$ and $\theta_{1 w_{2}}$ are constants.

Energy equation (53), in dimensionless form with the help of (7) and (12), becomes

$$
\frac{d^{2} T}{d \eta^{2}}=G P_{r} u-P_{r} E_{r}\left[\left\{\left(\frac{d u}{d \eta}\right)^{2}+\left(\frac{d v}{d \eta}\right)^{2}\right\}+M^{2}\left\{\left(\frac{d H_{x}}{d \eta}\right)^{2}+\left(\frac{d H_{y}}{d \eta}\right)^{2}\right\}\right],
$$

where $T=g \beta^{\prime} L^{3}\left(\theta_{1}-\theta_{1 w_{1}}\right) / v^{2}$ is dimensionless fluid temperature. $P_{r}=\rho v c_{p} / k$ is Prandtl number and $E_{r}=g \beta^{\prime} L / c_{p}$ is Eckert number.

Boundary conditions (54), in dimensionless form, become

$\mathrm{T}=0$ at $\eta=1, T=g \beta^{\prime} L^{3}\left(\theta_{1 w_{2}}-\theta_{1 w_{1}}\right) / v^{2}=\theta_{0}$ (say) at $\eta=-1$.

For solving eqn. (55), the eqn. (55) is combined with the following equation

$$
G P_{r} v=\frac{d^{2} \psi}{d \eta^{2}}
$$

with boundary conditions

$$
\psi=0 \text { at } \eta= \pm 1
$$

where $\Delta=T+i \psi$.

The resulting equation is presented as

$$
\frac{d^{2} \Delta}{d \eta^{2}}=G P_{r} F-P_{r} E_{r}\left[\left(\frac{d F}{d \eta} \frac{d \bar{F}}{\eta}\right)+M^{2}\left(\frac{d H}{d \eta} \frac{d \bar{H}}{d \eta}\right)\right],
$$

where $\bar{F}$ and $\bar{H}$ are complex conjugates of $F$ and $H$ respectively.

Boundary conditions (56) and (58), in combined form, become

$$
\Delta=0 \text { at } \eta=1, \Delta=\theta_{0} \text { at } \eta=-1 .
$$

The numerical solution of energy equation (59) subject to the boundary conditions (60) can be obtained by MATLAB software. The numerical values of rate of heat transfer at both the plates are obtained with the help of MATLAB software.

\section{Results and Discussion}

To study the effects of buoyancy force, rotation, magnetic field and wall conductance on the fluid velocity and induced magnetic field profiles of the velocity and induced magnetic field are drawn versus channel width variable $\eta$ in Figs. 2 to 10 for various values of $G, K^{2}, M^{2}, \phi_{1}$ and $\phi_{2}$ taking $R=-1$. It is evident from Figs. 2 to 10 that profiles of the velocity and induced magnetic field are asymmetric and there arises reverse flow in the primary and secondary flow directions due to the presence of free convection $(G \neq 0)$. It is evident from Fig. 2 that free convection tends to accelerate fluid flow in the primary flow directions whereas it accelerates secondary flow in the lower half of the channel and its characteristics are changed in the upper half of the channel. Fig. 3 reveals that free convection has tendency to increase primary as well as secondary induced magnetic fields $H_{x}$ and 
$H_{y}$ throughout the channel. Fig. 4 shows that rotation retards fluid flow in both the directions in the region away from the upper plate whereas, in the region near the upper plate, its characteristics are changed due to flow reversal.

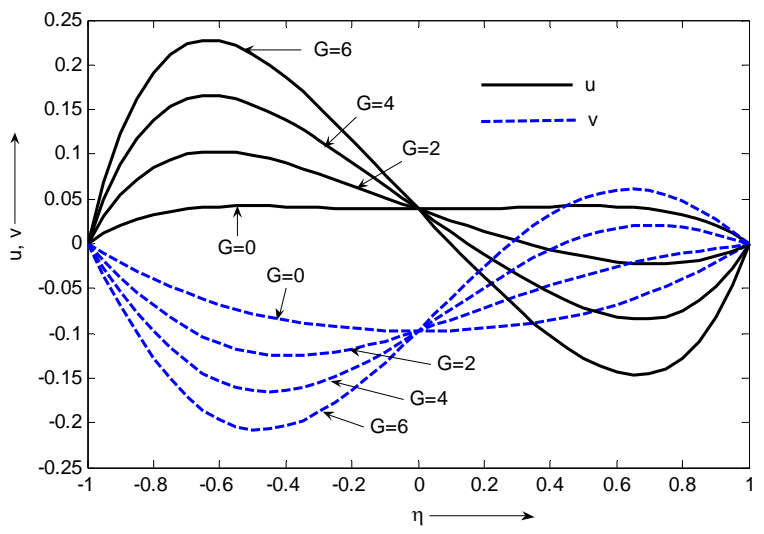

Fig. 2. Profiles of primary and secondary velocities when $K^{2}=5, M^{2}=4$ and $\phi_{1}=\phi_{2}=1$.

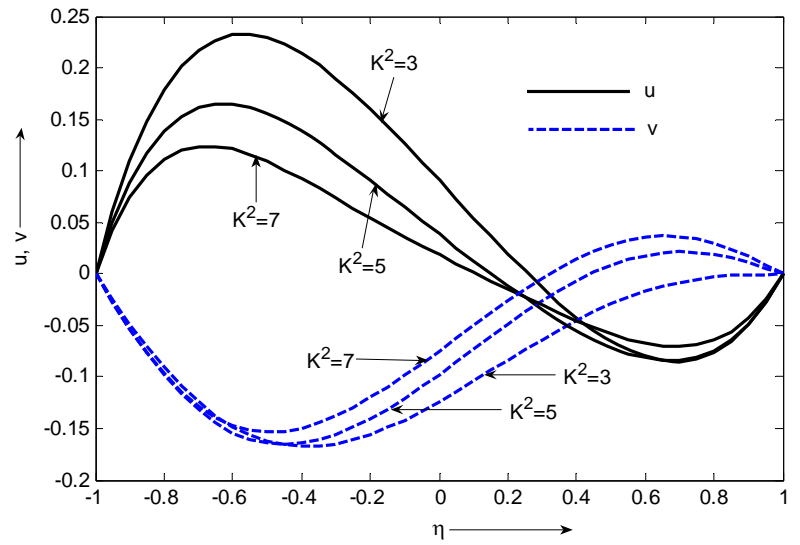

Fig. 4. Profiles of primary and secondary velocities when $G=4, M^{2}=4$ and $\phi_{1}=\phi_{2}=1$.

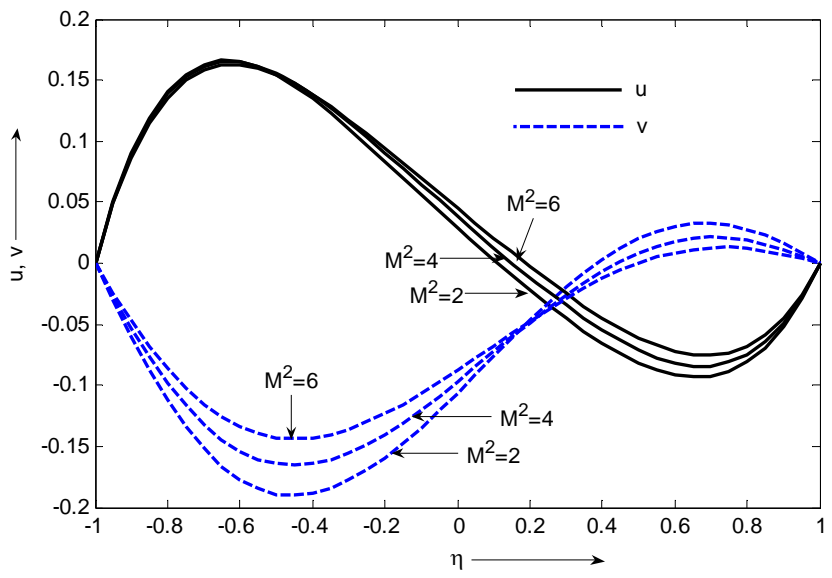

Fig. 6. Profiles of primary and secondary velocities when $G=4, K^{2}=5$ and $\phi_{1}=\phi_{2}=1$.

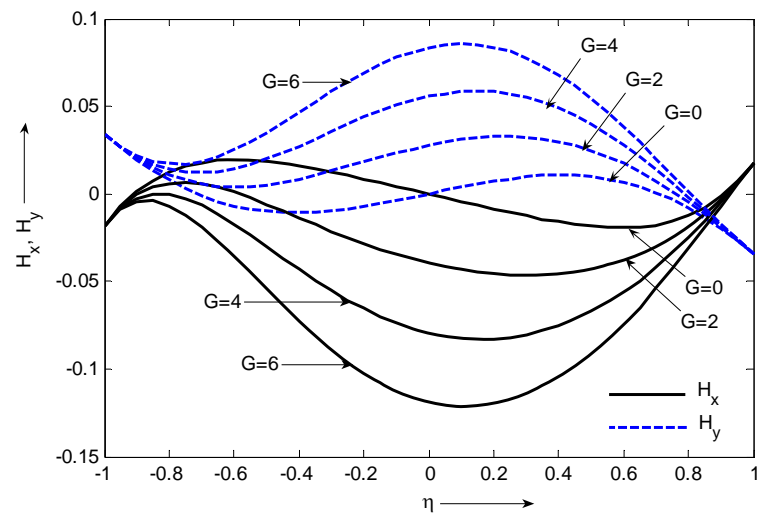

Fig. 3. Profiles of primary and secondary induced magnetic fields when $K^{2}=5, M^{2}=4$ and $\phi_{1}=\phi_{2}=1$.

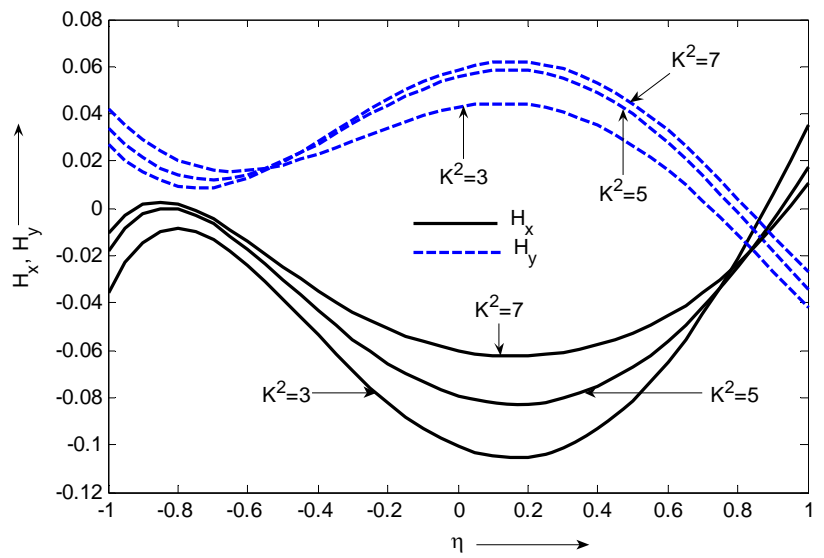

Fig. 5. Profiles of primary and secondary induced magnetic fields when $G=4, M^{2}=4$ and $\phi_{1}=\phi_{2}=1$.

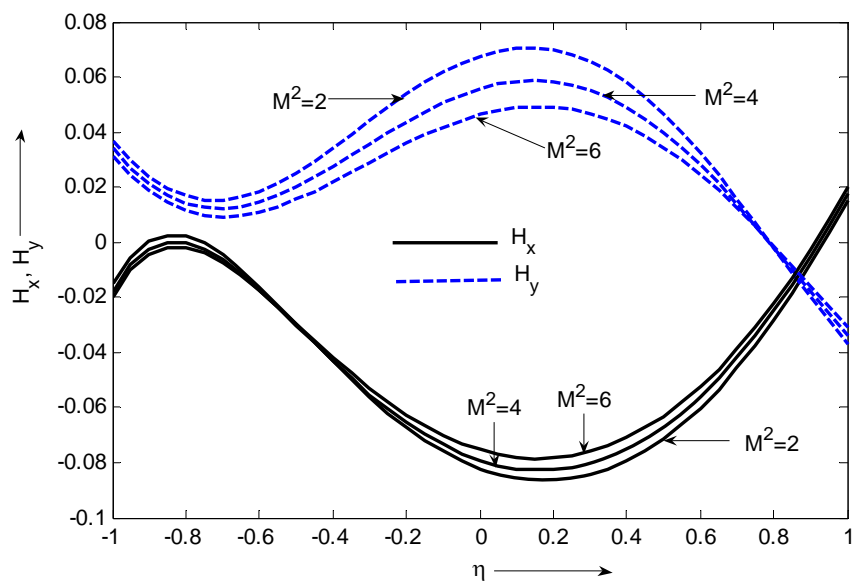

Fig. 7. Profiles of primary and secondary induced magnetic fields when $G=4, K^{2}=5$ and $\phi_{1}=\phi_{2}=1$. 


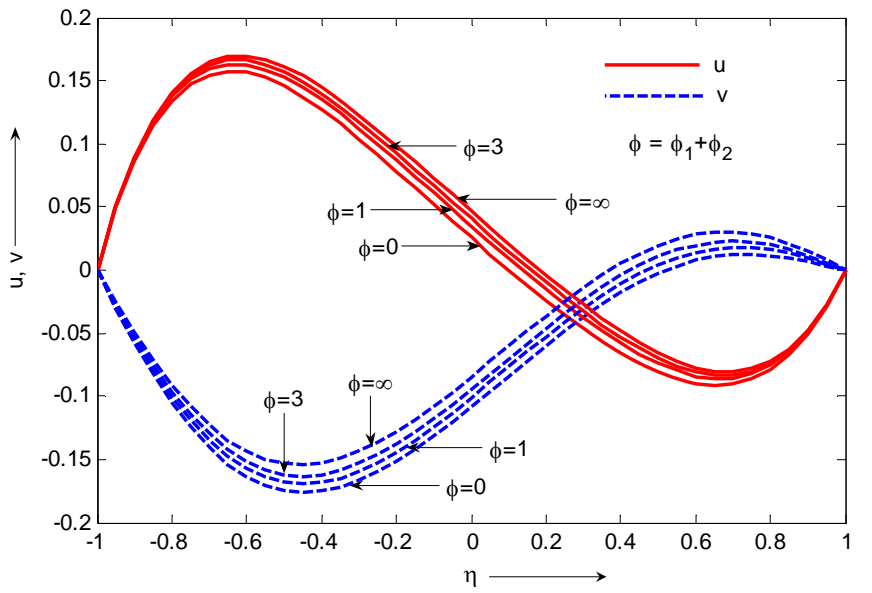

Fig. 8. Profiles of primary and secondary velocities when $G=4, K^{2}=5$ and $M^{2}=4$.

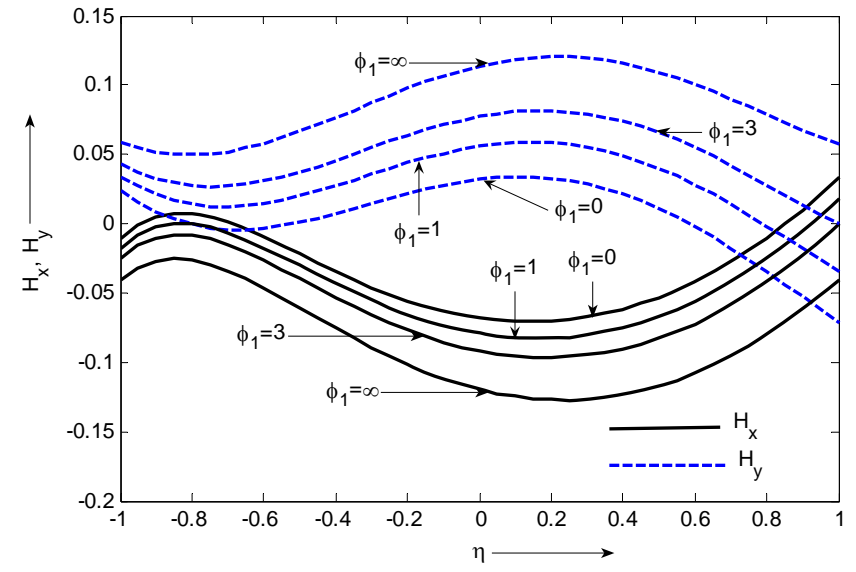

Fig. 9. Profiles of primary and secondary induced magnetic fields when $G=4, K^{2}=5, M^{2}=4$ and $\phi_{2}=1$.

It is noticed from Fig. 5 that rotation reduces primary induced magnetic field $H_{x}$ whereas it tends to increase secondary induced magnetic field $H_{y}$. However, characteristics of $H_{x}$ near the upper plate and of $H_{y}$ near the lower plate are changed due to rotation. Fig. 6 shows that magnetic field retards fluid flow in both the directions. Fig. 7 reveals that magnetic field reduces primary and secondary induced magnetic fields. However, characteristics of $H_{x}$ is changed near the upper plate and that of $H_{y}$ is changed near the lower plate of the channel. It is evident from Fig. 8 that, with the increase in $\phi=\phi_{1}+\phi_{2}$, primary velocity $u$ increases whereas secondary velocity $v$ decreases. This implies that wall conductance tends to accelerates fluid flow in the primary flow direction and has tendency to retard fluid flow in secondary flow direction. It is also noticed from Fig. 8 that primary velocity $\mathrm{u}$ is minimum when channel walls are non-conducting (i.e. $\phi=0)$ and it is maximum when the channel walls are perfectly conducting (i.e. $\phi=\infty)$. Secondary velocity $v$ is maximum for non-conducting walls and it is minimum for perfectly conducting walls. Figs. 9 and 10 show that both the primary and secondary induced magnetic fields increase with the increase in $\phi_{1}$ whereas it decrease with the increase in $\phi_{2}$. This implies that wall conductance of lower plate increases both the induced magnetic fields whereas wall conductance of the upper plate reduces both the induced magnetic fields.

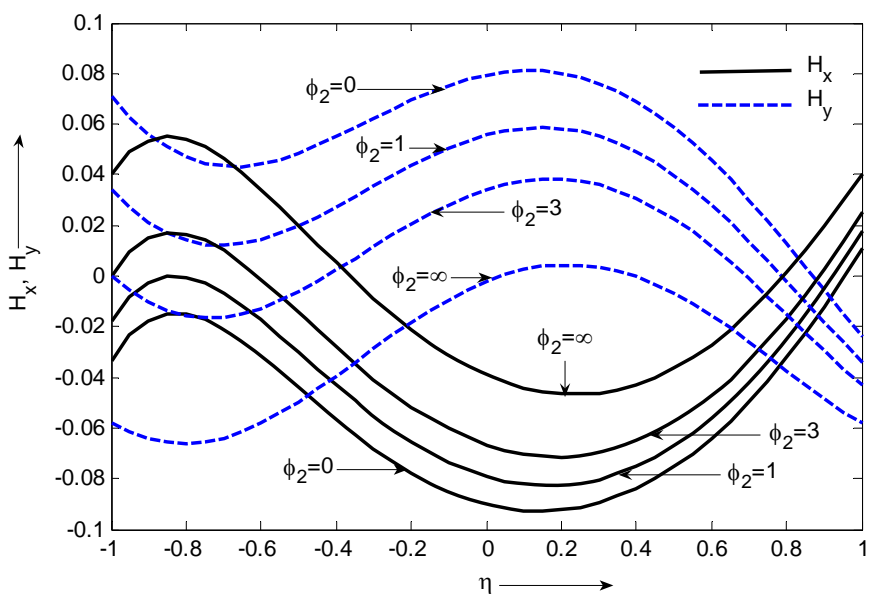

Fig. 10. Profiles of primary and secondary induced magnetic fields when $G=4, K^{2}=5$

$$
M^{2}=4 \text { and } \phi_{1}=1 \text {. }
$$

The numerical values of primary and secondary shear stress components at the upper plate are presented in tabular form in Tables 1 and 2 for various values of $M^{2}, K^{2}, G$ and $\phi$ taking $R=-1$ whereas that of critical Grashof numbers $G_{c x}$ and $G_{c y}$ for which separation takes place at the upper plate in the primary and secondary flow directions respectively and mass flow rates $Q_{x} / \rho v$ and $Q_{y} / \rho v$ in the primary and secondary flow directions respectively are provided in Tables 3 to 6 for various values of $M^{2}, K^{2}$ and $\phi$. It is evident from the Tables 1 and 2 that the primary shear stress $\tau_{x}$ at the upper plate increases with the increase 
in $G$ whereas secondary shear stress $\tau_{y}$ at the upper plate decreases, attains a minimum and then increases in magnitude with $G$ which implies that there exists flow separation at the upper plate due to free convection in the secondary flow direction. Primary shear stress $\tau_{x}$ increases, attains a maximum and then decreases with the increase in $K^{2}$ when $G=2$ whereas it decreases on increasing $K^{2}$ when $G=4$ and 6 ,. On increasing $K^{2}$, secondary shear stress $\tau_{y}$ decreases when $G=2$ and it increases in magnitude when

Table 1. Primary and secondary shear stress components at the upper plate $\eta=1$ when $M^{2}=4$ and $\phi=1$.

\begin{tabular}{|c|c|c|c|c|c|c|}
\hline \multicolumn{5}{|c|}{$\tau_{\mathrm{x}}$} & \multicolumn{3}{c|}{$\tau_{\mathrm{y}}$} \\
\hline$G_{\downarrow} K_{\rightarrow}^{2}$ & 3 & 5 & 7 & 3 & 5 & 7 \\
\hline 2 & 0.1367 & 0.1905 & 0.1860 & 0.1675 & 0.0723 & 0.0165 \\
\hline 4 & 0.6266 & 0.6239 & 0.5679 & 0.0454 & -0.0922 & -0.1650 \\
\hline 6 & 1.1165 & 1.0573 & 0.9498 & -0.0767 & -0.2566 & -0.3465 \\
\hline
\end{tabular}

Table 2. Primary and secondary shear stress components at the upper plate $\eta=1$ when $K^{2}=5$ and $G=4$.

\begin{tabular}{|c|c|c|c|c|c|c|}
\hline \multicolumn{5}{|c|}{$\tau_{\mathrm{x}}$} & \multicolumn{3}{|c|}{$-\tau_{\mathrm{y}}$} \\
\hline$\phi_{\downarrow} \quad M^{2} \rightarrow$ & 2 & 4 & 6 & 2 & 4 & 6 \\
\hline 0 & 0.6695 & 0.6395 & 0.6049 & 0.1408 & 0.0586 & 0.0093 \\
\hline 1 & 0.6611 & 0.6239 & 0.5835 & 0.1567 & 0.0922 & 0.0433 \\
\hline 3 & 0.6558 & 0.6168 & 0.5778 & 0.1695 & 0.1186 & 0.0829 \\
\hline$\infty$ & 0.6500 & 0.6134 & 0.5823 & 0.1885 & 0.1555 & 0.1333 \\
\hline
\end{tabular}

$G=6$. Also $\tau_{y}$ decreases, attains a minimum and then increases in magnitude with the increase in $K^{2}$ when $G=4$. $\tau_{x}$ and $\tau_{y}$ decrease on increasing $M^{2} . \tau_{x}$ decreases with the increase in $\phi$ when $M^{2}=2$ and 4 and it decreases, attains a minimum and then increases with the increase in $\phi$ when $M^{2}=6 . \tau_{y}$ increases on increasing $\phi$. It is found from Tables 3 and 4 that critical Grashof number $G_{c x}$, for which separation takes place at the upper plate in the primary flow direction, and critical Grashof number $G_{c y}$, for which separation takes place at the upper plate in the secondary flow direction, decrease on increasing either $K^{2}$ or $\phi$ which implies that rotation and wall conductance induce flow separation at the upper plate in both the primary and secondary flow directions. $G_{c x}$ and $G_{c y}$ are minimum when $\phi=\infty$ (i.e. perfectly conducting walls) and these are maximum when $\phi=0$ (i.e. non-conducting walls). This implies that separation takes place early at the upper plate in both the directions for perfectly conducting walls. $G_{c x}$ and $G_{c y}$ increase with the increase in $M^{2}$ which implies that magnetic field prohibits flow separation at the upper plate in both the directions i.e. it suppresses any turbulence present in the fluid flow to maintain laminar flow characteristics of the fluid. It is observed from the Tables 5 and 6 that mass flow rate $Q_{x} / \rho v$ in the primary flow direction increases with the increase in either $M^{2}$ or $\phi$ and it decreases with the increase in $K^{2}$. Mass flow rate $Q_{y} / \rho v$ in the secondary flow direction decreases with the increase in either $M^{2}$ or $K^{2}$ or $\phi$.

Table 3. Critical Grashof numbers at the upper plate $\eta=1$ when $M^{2}=4$.

\begin{tabular}{|c|c|c|c|c|c|c|}
\hline \multicolumn{5}{|c|}{$\mathrm{G}_{\mathrm{cx}}$} & \multicolumn{3}{|c|}{$-\mathrm{G}_{\mathrm{cy}}$} \\
\hline$\phi_{\downarrow} \quad K_{\rightarrow}^{2} \rightarrow$ & 3 & 5 & 7 & 3 & 5 & 7 \\
\hline 0 & 1.6538 & 1.3306 & 1.2297 & 1.0778 & 0.7425 & 0.5536 \\
\hline 1 & 1.6350 & 1.3423 & 1.2388 & 0.7749 & 0.5832 & 0.4481 \\
\hline 3 & 1.5762 & 1.3306 & 1.2350 & 0.5770 & 0.4655 & 0.3672 \\
\hline$\infty$ & 1.4553 & 1.2878 & 1.2139 & 0.3525 & 0.3116 & 0.2555 \\
\hline
\end{tabular}


Table 4. Critical Grashof numbers at the upper plate $\eta=1$ when $\phi=1$.

\begin{tabular}{|c|c|c|c|c|c|c|}
\hline \multicolumn{5}{|c|}{$\mathrm{G}_{\mathrm{cx}}$} & \multicolumn{3}{c|}{$-\mathrm{G}_{\mathrm{cy}}$} \\
\hline$M_{\downarrow}^{2} K_{\rightarrow}^{2}$ & 3 & 5 & 7 & 3 & 5 & 7 \\
\hline 2 & 1.4816 & 1.2559 & 1.1902 & 0.7315 & 0.5021 & 0.3723 \\
\hline 4 & 1.6350 & 1.3423 & 1.2388 & 0.7749 & 0.5832 & 0.4481 \\
\hline 6 & 1.7717 & 1.4313 & 1.2927 & 0.7916 & 0.6477 & 0.5153 \\
\hline
\end{tabular}

Table 5. Mass flow rates in the primary and secondary flow directions when $M^{2}=4$.

\begin{tabular}{|c|c|c|c|c|c|c|}
\hline \multicolumn{5}{|c|}{$\mathrm{Q}_{\mathrm{x}} / \rho v$} & \multicolumn{3}{c|}{$-\mathrm{Q}_{\mathrm{y}} / \rho v$} \\
\hline$\phi_{\downarrow} \quad K_{\rightarrow}^{2}$ & 3 & 5 & 7 & 3 & 5 & 7 \\
\hline 0 & 0.0608 & 0.0270 & 0.0155 & 0.1101 & 0.0773 & 0.0582 \\
\hline 1 & 0.0688 & 0.0332 & 0.0194 & 0.0925 & 0.0713 & 0.0556 \\
\hline 3 & 0.0714 & 0.0369 & 0.0221 & 0.0796 & 0.0661 & 0.0532 \\
\hline$\infty$ & 0.0714 & 0.0407 & 0.0254 & 0.0634 & 0.0584 & 0.0493 \\
\hline
\end{tabular}

Table 6. Mass flow rates in the primary and secondary flow directions when $\phi=1$.

\begin{tabular}{|c|c|c|c|c|c|c|}
\hline \multicolumn{5}{|c|}{$\mathrm{Q}_{\mathrm{x}} / \rho v$} & \multicolumn{3}{c|}{$-\mathrm{Q}_{\mathrm{y}} / \rho v$} \\
\hline$M_{\downarrow}^{2} K_{\rightarrow}^{2}$ & 3 & 5 & 7 & 3 & 5 & 7 \\
\hline 2 & 0.0624 & 0.0285 & 0.0166 & 0.1048 & 0.0750 & 0.0570 \\
\hline 4 & 0.0688 & 0.0332 & 0.0194 & 0.0925 & 0.0713 & 0.0556 \\
\hline 6 & 0.0721 & 0.0370 & 0.0220 & 0.0809 & 0.0671 & 0.0539 \\
\hline
\end{tabular}

The numerical values of rate of heat transfer at the upper and lower plates i.e. $(d T / d \eta)_{\eta=1}$ and $(d T / d \eta)_{\eta=-1}$, respectively, are presented in Tables 7 and 8 for various values of $M^{2}, K^{2}, G$ and $\phi$ taking $R=-1, P_{r}=0.025$ and $E_{r}=2$. It is evident from Tables 7 and 8 that rate of heat transfer at the upper plate $(d T / d \eta)_{\eta=1}$ increases with the increase in $G$ and decreases with the increase in either $K^{2}$ or $\phi .(d T / d \eta)_{\eta=1}$ increases with the increase in $M^{2}$ when $\phi \neq \infty$ and it decreases on increasing $M^{2}$ when $\phi=\infty$. Rate of heat transfer at the lower plate i.e. $(d T / d \eta)_{\eta=-1}$ decreases with the increase in $G$ and increases with the increase in either $K^{2}$ or $\phi .(d T / d \eta)_{\eta=-1}$ decreases with the increase in $M^{2}$ when $\phi=0$ and increases with the increase in $M^{2}$ when $\phi \neq 0$. Thus we conclude that free convection tends to increase rate of heat transfer at the upper plate whereas it has reverse effects on the rate of heat transfer at the lower plate. Rotation and wall conductance have tendency to reduce rate of heat transfer at the upper plate whereas it has reverse effects on the rate of heat transfer at the lower plate. Magnetic field tends to increase rate of heat transfer at the upper plate when both the plates are either non-conducting or finitely conducting and it has tendency to reduce rate of heat transfer at the lower plate when both the plates are either finitely conducting or perfectly conducting. 
Table 7. Rate of heat transfer at the upper and lower plates when $M^{2}=4$ and $\phi=2$.

\begin{tabular}{|c|c|c|c|c|c|c|}
\hline \multicolumn{5}{|c|}{$-(\mathrm{d} \theta / \mathrm{d} \eta)_{/ \eta=1}$} & \multicolumn{3}{c|}{$-(\mathrm{d} \theta / \mathrm{d} \eta)_{/ \eta=-1}$} \\
\hline$G_{\downarrow} \quad K_{\rightarrow}^{2}$ & 3 & 5 & 7 & 3 & 5 & 7 \\
\hline 2 & 0.5058 & 0.5045 & 0.5034 & 0.4946 & 0.4961 & 0.4970 \\
\hline 4 & 0.5137 & 0.5120 & 0.5096 & 0.4928 & 0.4936 & 0.4948 \\
\hline 6 & 0.5299 & 0.5262 & 0.5210 & 0.4881 & 0.4889 & 0.4907 \\
\hline
\end{tabular}

Table 8. Rate of heat transfer at the upper and lower plates when $K^{2}=5$ and $G=4$.

\begin{tabular}{|c|c|c|c|c|c|c|}
\hline \multicolumn{5}{|c|}{$-(\mathrm{d} \theta / \mathrm{d} \eta)_{/ \eta=1}$} & \multicolumn{3}{c|}{$-(\mathrm{d} \theta / \mathrm{d} \eta)_{/ \eta=-1}$} \\
\hline$\phi_{\downarrow} M_{\rightarrow}^{2}$ & 2 & 4 & 6 & 2 & 4 & 6 \\
\hline 0 & 0.5119 & 0.5141 & 0.5159 & 0.4926 & 0.4924 & 0.4918 \\
\hline 1 & 0.5108 & 0.5120 & 0.5128 & 0.4932 & 0.4936 & 0.4939 \\
\hline 3 & 0.5102 & 0.5107 & 0.5110 & 0.4935 & 0.4942 & 0.4948 \\
\hline$\infty$ & 0.5094 & 0.5094 & 0.5093 & 0.4937 & 0.4947 & 0.4954 \\
\hline
\end{tabular}

\section{Conclusions}

Combined free and forced convection flow of a viscous incompressible electrically conducting fluid in a rotating channel is investigated. Asymptotic behavior of the solution for the velocity and induced magnetic field is analyzed for large values of $M^{2}$ and $K^{2}$ to gain some physical insight into the flow pattern. Free convection causes reverse flow in the primary and secondary flow directions and tends to accelerate primary flow and has tendency to accelerate secondary flow in the lower half of the channel. Free convection tends to increase primary and secondary induced magnetic fields throughout the channel. Magnetic field retards fluid flow in both the directions. Wall conductance accelerates fluid flow in the primary flow direction and it retards fluid flow in the secondary flow direction. There exists flow separation at the upper plate due to free convection in the secondary flow direction. Free convection tends to increase rate of heat transfer at the upper plate whereas it has reverse effects on the rate of heat transfer at the lower plate. Rotation and wall conductance tend to reduce rate of heat transfer at the upper plate and have reverse effects on the rate of heat transfer at the lower plate.

Acknowledgement: Authors are grateful to referees for their valuable suggestions and comments for the improvement of this research paper.

\section{References}

Acheson, D.J., 1975. Forced hydromagnetic oscillations of a rapidly rotating fluid, Phys. Fluids, Vol. 18, pp. 961.

Das, S., Maji, S.L., Guria, M. and Jana, R.N., 2009. Unsteady MHD Couette flow in a rotating system, Math. Comp. Modelling, Vol. 50, p. 1211.

Datta, N. and Jana, R.N., 1977. Hall effects on hydromagnetic flow and heat transfer in a rotating channel, J. Inst. Math. Applics., Vol. 19, pp. 217.

Debnath, L., 1974. On Ekman and Hartmann boundary layers in a rotating fluid, Acta Mech., Vol. 18, pp. 333.

Debnath, L., 1975. Resonant oscillations of a porous plate in an electrically conducting rotating viscous fluid, Phys. Fluids, Vol. 17, pp. 1704.

Ghosh, S.K., 1993. Unsteady hydromagnetic flow in a rotating channel with oscillating pressure gradient, J. Phy. Soc. Japan, Vol. 62, No. 11, pp. 3893.

Ghosh, S.K., and Bhattacharjee, P.K., 2000. Magnetohydrodynamic convective flow in a rotating channel, Archives of Mech., Vol. 52, pp. 303.

Ghosh, S.K. and Pop, I., 2003. Hall effects on unsteady hydromagnetic flow in a rotating system with oscillating pressure gradient, Int. J. Appl. Mech. Eng., Vol. 8, pp. 43. 
Ghosh, S.K. and Pop, I., 2004. Hall effects on MHD plasma Couette flow in a rotating environment, Int. J. Appl. Mech. Eng., Vol. 9, p. 293.

Guria, M., Das, S., Jana, R.N. and Ghosh, S.K., 2009. Oscillatory Couette flow in the presence of an inclined magnetic field, Meccanica, Vol. 44, p. 555.

Hayat, T., Nadeem, S. and Asghar, S., 2004. Hydromagnetic Couette flow of an Oldroyd-B fluid in a rotating system, Int. J. Engng. Sci., Vol. 42, pp. 65.

Hayat, T., Nadeem, S., Siddiqui, A.M. and Asghar, S., 2004. An oscillating hydromagnetic non-newtonian flow in a rotating system, Appl. Math. Lett., Vol. 17, pp. 609.

Hayat, T., Wang, Y. and Hutter, K., 2004. Hall effects on the unsteady hydromagnetic oscillatory flow of a second-grade fluid, Int. J. Non-Linear Mech., Vol. 39, pp. 1027.

Hide, R. and Roberts, P.H., 1960. Hydromagnetic flow due to an oscillating plane, Rev. Mod. Phys., Vol. 32, pp. 799.

Mazumder, B.S., 1977. Effect of wall conductances on hydromagnetic flow and heat transfer in a rotating channel, Acta Mech., Vol. 28, pp. 85 .

Nagy, T., and Demendy, Z., 1995. Effects of Hall current and Coriolis force on Hartmann flow under general wall conditions, Acta Mech., Vol. 113, pp. 77.

Nanda, R.S. and Mohanty, H.K., 1971. Hydromagnetic flow in a rotating channel, Appl. Sci. Res., Vol. 24, pp. 65.

Raptis, A. and Singh, A.K., 1986. Hydromagnetic Rayleigh problem in a rotating fluid, Acta Physica Hungarica, Vol. 60, pp. 221.

Seth, G.S., Jana, R.N. and Maiti, M.K., 1982. Unsteady hydromagnetic Couette flow in a rotating system, Int. J. Engng. Sci., Vol. 20, pp. 989.

Seth, G.S., and Banerjee, B., 1996, Hydromagnetic convective flow in a rotating channel" Proc. Math. Soc., BHU, Varanasi, India, Vol. 12, pp. 47.

Seth, G.S. and Singh,M.K., 2008. Combined free and forced convection MHD flow in a rotating channel with perfectly conducting walls, Ind. J. Theo. Phys., Vol. 56, pp. 203.

Seth, G.S. and Ansari, Md. S., 2009. Magnetohydrodynamic convective flow in a rotating channel with Hall effects, Int. J. Theor. Appl. Mech., Vol. 4, No. 2, pp. 205.

Seth, G.S., Ansari, Md. S. and Nandkeolyar, R., 2010. Unsteady hydromagnetic couette flow within porous plates in a rotating system, Adv. in Appl. Math. Mech., Vol. 2, No. 3, pp. 286.

Seth, G.S., Nandkeolyar, R., Mahto, N. and Singh, S.K., 2009. MHD Couette flow in a rotating system in the presence of inclined magnetic field, Appl. Math. Sci., Vol. 3, pp. 2919.

Singh, A.K., Sacheti, N.C. and Chandran, P., 1994. Transient effects on Magnetohydrdynamic Couette flow with rotation, Int. J. Engng. Sci., Vol. 32, pp. 133.

Singh, K.D., 2000. An oscillatory hydromagnetic Couette flow in a rotating system, ZAMM, Vol. 80, pp. 429.

Soundalgekar, V.M. and Pop, I., 1973. On hydromagnetic flow in a rotating fluid past an infinite porous wall, ZAMM, Vol. 53, pp. 718.

Soundalgekar, V.M., and Gupta, A.S., 1975. On hydromagnetic Flow and heat transfer in a rotating fluid past an infinite porous wall, ZAMM, Vol. 55, pp. 762.

\section{Biographical notes}

Dr. G.S. Seth is an Associate Professor in the Department of Applied Mathematics, Indian School of Mines, Dhanbad, India. He received his Ph.D. degree in Mathematics from Indian Institute of Technology, Kharagpur, India. He has more than twenty nine years of experience in teaching and research. His current area of research studies includes Fluid dynamics, Magnetohydrodynamics and heat transfer. He was visiting Assistant Professor at University of Aden, Republic of Yemen during the period September 01, 1991 to August 31, 1993. He has published more than forty two research papers in national/international journals of repute.

Dr. N. Mahto is an Associate Professor in the Department of Mathematics, R.S.P. College, Jharia, Dhanbad of Vinoba Bhave University, Hazaribag, India. He received his Ph.D. degree in Applied Mathematics from Indian School of Mines, Dhanbad, India. He has more than twenty eight years of teaching and research experience. He has published eleven research papers in national/international journals of repute.

Md. S. Ansari received M.Sc. degree in Mathematics and Computing from Indian School of Mines, Dhanbad, India and subsequently joined the department of Applied Mathematics of the same institute to do the research work leading to Ph.D. degree on the topic entitled "Theoretical Study of Hydromagnetic Flow of a Viscous Incompressible Electrically Conducting Fluid in Non-Rotating and Rotating Media". He has already submitted his Ph.D. thesis to Indian School of Mines, Dhanbad, India in February, 2010. He has four years of research experience. He has published eleven research papers in national/ international journals of repute.

R. Nandkeolyar received M.Sc. degree in Mathematics from Vinoba Bhave University, Hazaribag, India in the year 2005 and M.Phil. degree in Applied Mathematics from Indian School of Mines, Dhanbad, India in the year 2007. Subsequently he joined the department of Applied Mathematics, Indian School of Mines, Dhanbad, India as a Junior Research Fellow to do research work leading to Ph.D. degree on the topic entitled "An Investigation of Some Problems of Magnetohydrodynamic Flow and Heat Transfer". He has three and half years of research experience and has published eight research papers in journals of national/international repute.

Received June 2010

Accepted July 2010

Final acceptance in revised form August 2010 